Textures and Microstructures, Vol. 34, pp. 233-241 Reprints available directly from the publisher Photocopying permitted by license only
(C) 2000 OPA (Overseas Publishers Association) N.V. Published by license under the Gordon and Breach Science Publishers imprint. Printed in Malaysia.

\title{
$r$-DEPENDENCE IN TWO-POINT ORIENTATION COHERENCE FUNCTIONS
}

\author{
PETER R. MORRIS \\ 1276 Oakmont Dr., Hamilton OH 45013, USA
}

(Received 12 August 1999; In final form 8 April 2000)

Functions are derived, which are orthonormal on the range $r=0,1$, with weight function corresponding to the distribution of $r$ in a typical experimental procedure for measurement of the two-point orientation-coherence (or orientation-correlation) function. These are obtained by making an appropriate change of variable in spherical Bessel functions, orthonormal on the range $r=0,1$, with unit weight function. The effects of weight function and change of variable on the functions are considered.

Keywords: Orientation coherence function; Spherical Bessel functions; Orthonormality

\section{INTRODUCTION}

The two-point orientation-coherence (or orientation-correlation) function (OCF), $f\left(g, r, g^{\prime}\right)$, describes the probability that $g$ and $g^{\prime}$ are orientations associated with the end points of a vector $r$, randomly located within a polycrystal. This function is similar to the two-point probability density described by Kröner (1986). Morris et al. (1988) and Wang (1988) have suggested series representations for the OCF. Following Bunge (1965) and Roe (1965), generalized spherical harmonics are used to describe $g$ and $g^{\prime}$. Spherical surface harmonics seem appropriate for representing the angular dependence of $r$. The choice of functions for representation of $r=|r|$ is less obvious. Wang (1988) suggested $r^{\rho}$. These functions are not orthogonal, nor wellbehaved for increasing $r$. Morris et al. (1988) suggested combinations of $\mathrm{e}^{-\alpha r} \sin (j r)$. These functions require quadruple-precision computer 
calculations, and may not be complete. It has been previously noted that, in any finite-volume experiment, small $r$ occur with greatest frequency. A typical experiment (Wang, 1988, p. 35) involves ElectronBackscattering Diffraction (EBD) from a series of circular sections taken through successive longitude meridians.

\section{WEIGHT FUNCTION}

If we denote the longitude of the vector $r$ by $\eta$, and the colatitude by $\chi, \eta$ is determined by the angle of $x^{\prime}$ from the $x$ toward the $y$ sample direction of the particular section, whose surface is determined by $x^{\prime}$ and $z . \chi$ is determined by the angle from the common $z$ axis to the vector $r . x, y$ and $z$ are presumed uniquely defined by sample symmetry, rolling, transverse and normal directions, e.g., Figure 1 illustrates the relation of $\eta, x^{\prime}, \chi$ and $r$ to the $x, y$ and $z$ sample coordinates.

For given $\eta, \chi$, we seek to characterize $g$ and $g^{\prime}$ as a function of $r$. To do this, we inscribe a square grid, within a circular section of longitude $\eta$, with lines perpendicular and parallel to the direction of $r$ specified by $\eta$ and $\chi$. We may consider circular sections of unit diameter, without loss of generality. The results may subsequently be scaled to conform to actual dimension. We consider a vector of length $r$, where $0 \leq r \leq 1$. We consider one-fourth of the circle, bounded by radii perpendicular and parallel to $r$. Initially the head of the vector $r$ is placed at $X_{i}, Y_{i}$, where $r$ is parallel to the $Y$ axis, and $Y_{i}=r / 2$. (We consider only vectors of length corresponding to an integral multiple of the distance between successive grid points.) $X$ and $Y$ are local coordinates corresponding to a particular choice of $\eta$ and $\chi$ and should not be confused with $x$ and $y$ sample coordinates. For given $r$, we wish to determine the number of grid points which the head of the vector $r$ may occupy within the quarter circle. In this process, we restrict $Y_{i}$ to grid points $\geq r / 2$. $\left(Y_{i}<r / 2\right.$ can be considered by equivalent movement of the tail of the vector $r$ in the lower quarter circle.) Figure 2 illustrates a square grid with $X$ and $Y$ perpendicular and parallel to $r$ inscribed in a circular section of unit diameter and one possible position of a vector $r$ of length 0.6 . If we introduce 


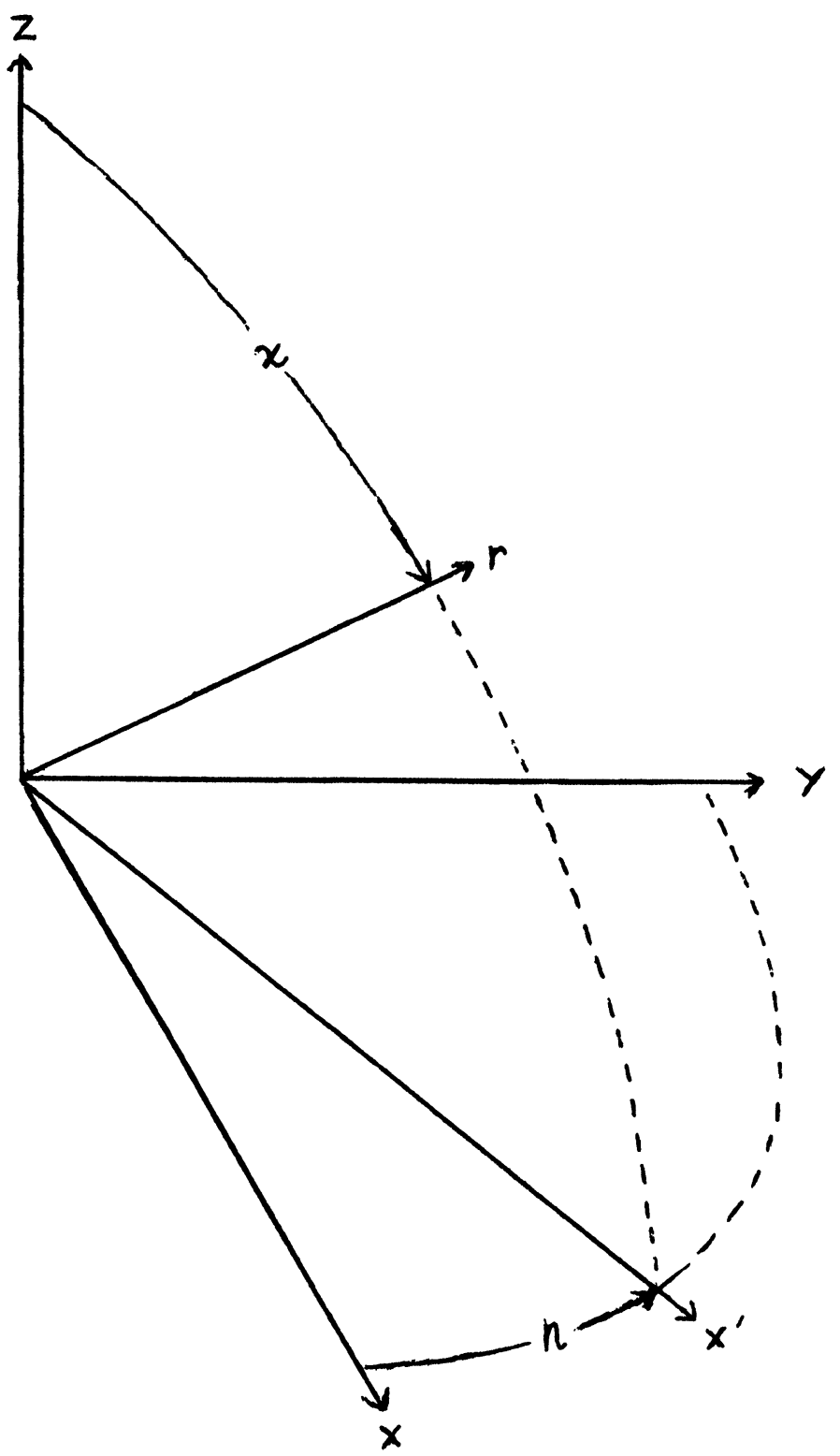

FIGURE 1 Illustration of longitude, $\eta$, measured from $x$ sample direction toward $y$ sample direction and colatitude, $\chi$, measured from $z$ sample direction. $\eta$ and $\chi$ determine the orientation of $r$. 


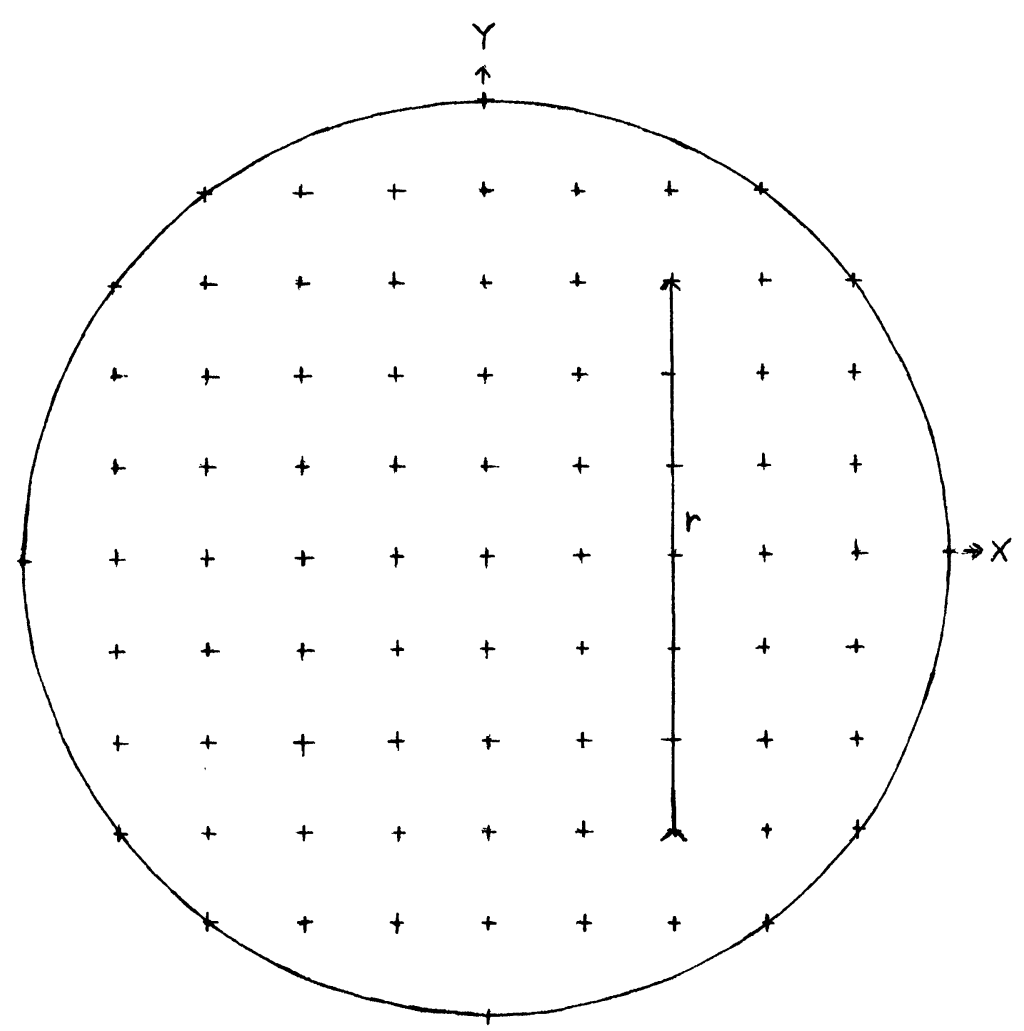

FIGURE 2 Square grid with $X$ and $Y$ perpendicular and parallel to $r$, inscribed in circular section of unit diameter, showing one possible position of a vector $r$ of length 0.6 .

successively finer grids, in the limit, where the separation between grid points becomes infinitesimal, the positions which the head of the vector $r$ may occupy within the quarter circle describe the area $A$ defined by $r / 2 \leq Y \leq 1 / 2$, i.e.,

$$
\begin{aligned}
A & =\int_{r / 2}^{1 / 2}\left(1 / 4-y^{2}\right)^{1 / 2} d y \\
& =\frac{1}{8}\left[\pi / 2-r\left(1-r^{2}\right)^{1 / 2}-\sin ^{-1}(r)\right] .
\end{aligned}
$$

The probability of finding a vector of length $r$ is thus proportional to the quantity in rectangular parenthesis. We seek to normalize this 
probability so that the integral over all possible $r$ is unity. To this end,

$$
\begin{aligned}
& \int_{0}^{1}\left[\pi / 2-r\left(1-r^{2}\right)^{1 / 2}-\sin ^{-1}(r)\right] d r \\
& \quad=\left[\pi r / 2+\left(1-r^{2}\right)^{3 / 2} / 3-r \sin ^{-1}(r)-\left(1-r^{2}\right)^{1 / 2}\right]_{0}^{1}=2 / 3 .
\end{aligned}
$$

Dividing the integrand by this result gives the probability, $P(r) d r$ of finding a vector of length between $r$ and $r+d r$ as

$$
P(r) d r=[3 / 2]\left[\pi / 2-r\left(1-r^{2}\right)^{1 / 2}-\sin ^{-1}(r)\right] d r .
$$

We desire functions, orthonormal on the range $r=0,1$, with weight function, $P(r)$, given by Eq. (3).

\section{SEARCH FOR FUNCTIONS}

Unable to find existing functions orthonormal with weight function, $P(r)$, given by Eq. (3), I have tried to directly construct such functions. Without exception, functions constructed in this manner tend to be illbehaved (become large) as $r$ approaches unity. It is also possible to employ a change of variable in functions orthonormal on the range $r=0,1$ with unit weight function, to produce functions orthonormal on the range $r=0,1$ with weight function $P(r)$, given by Eq. (3). If we set

$$
\begin{gathered}
d w=[3 / 2]\left[\pi / 2-r\left(1-r^{2}\right)^{1 / 2}-\sin ^{-1}(r)\right] d r, \text { then } \\
w=[3 / 2] \int\left[\pi / 2-r\left(1-r^{2}\right)^{1 / 2}-\sin ^{-1}(r)\right] d r+C \\
=3 \pi r / 4+\left(1-r^{2}\right)^{3 / 2} / 2-3\left(1-r^{2}\right)^{1 / 2} / 2-3 r \sin ^{-1}(r) / 2+C .
\end{gathered}
$$

Let $C=1$. Then $w=0$ when $r=0$, and $w=1$ when $r=1$. Thus, if we have a series of functions orthonormal on the range $r=0,1$ with unit weight function, replacing $r$ by $w$ and $d r$ by $d w$ yields a series of functions orthonormal on the range $r=0,1$ with the desired weight function. The spherical Bessel functions (Landau and Lifshitz, 1977) were chosen for this purpose. These can be expressed in terms of Bessel functions of half-integral order. We multiply the resultant functions by 
$r$ to produce functions orthogonal with unit weight function on the range $r=0,1$. The first few functions are:

$$
\begin{gathered}
R_{-1}(r)=-\sin (r)-\cos (r) / r=(\pi r / 2)^{1 / 2} J_{-3 / 2}(r) \\
R_{0}(r)=\cos (r)=(\pi r / 2)^{1 / 2} J_{-1 / 2}(r) \\
R_{1}(r)=\sin (r)=(\pi r / 2)^{1 / 2} J_{1 / 2}(r)
\end{gathered}
$$

and

$$
R_{2}(r)=\sin (r) / r-\cos (r)=(\pi r / 2)^{1 / 2} J_{3 / 2}(r)
$$

Subsequent functions may be obtained from the recurrence relation

$$
R_{i+1}(r)=(2 i-1) R_{i}(r) / r-R_{i-1}(r),
$$

(McLachlan, 1955), or the generating function

$$
R_{i}(r)=[-1]^{i-1} r^{i}\left[\frac{1}{r} \frac{d}{d r}\right]^{i-1}\left[\frac{\sin (r)}{r}\right],
$$

(Landau and Lifshitz, 1977). The integrals of products of these functions are given by McLachlan (1955).

$$
\int_{0}^{1} R_{i}(k r) R_{i}(l r) d r=0
$$

if $k$ and $l$ are different zeros of $R_{i}$, and

$$
\int_{0}^{1} R_{i}^{2}(k r) d r=\frac{1}{2} R_{i-1}^{2}(k)
$$

If we set

$$
R_{i j}(r)=2^{1 / 2} R_{i}\left(k_{j} r\right) /\left|R_{i-1}\left(k_{j}\right)\right|,
$$

where $k_{j}$ is the $j$ th zero of $R_{i}(r)$, we obtain a series of functions, orthonormal on the range $r=0,1$ with unit weight function. If, in 
these, we replace $r$ by $w$ from Eq. (5), we obtain a series of functions orthonormal on the range $r=0,1$, with weight function $P(r)$, Eq. (3).

\section{EFFECTS OF WEIGHT FUNCTION AND CHANGE OF VARIABLE}

Consider the expansion of a Dirac delta function, $\delta\left(r-r_{0}\right)$, in terms of the $R_{i j}(r)$ defined by Eqs. (6) to (14).

$$
\delta\left(r-r_{0}\right)=\sum_{j} b_{i j} R_{i j}\left(a_{j} w\right)
$$

where $a_{j}$ is the $j$ th zero of $R_{i}(r)$. Multipling both sides of Eq. (15) by $R_{i k}\left(a_{k} w\right) d w$, Eq. (4), gives

$$
\begin{aligned}
& \delta\left(r-r_{0}\right) R_{i k}\left(a_{k} w\right)[3 / 2]\left[\pi / 2-r\left(1-r^{2}\right)^{1 / 2}-\sin ^{-1}(r)\right] d r \\
& \quad=R_{i k}\left(a_{k} w\right) \sum_{j} b_{i j} R_{i j}\left(a_{j} w\right) d w
\end{aligned}
$$

where $d w$ and $w$ may be expressed in terms of $r$ from Eqs. (4) and (5). Integrating Eq. (16) with respect to $r$ from $r=0$ to $r=1$ yields

$$
R_{i k}\left(a_{k} w_{0}\right)[3 / 2]\left[\pi / 2-r_{0}\left(1-r_{0}^{2}\right)^{1 / 2}-\sin ^{-1}\left(r_{0}\right)\right]=\delta_{j k} b_{i j}=b_{i k},
$$

where $\delta_{j k}$ is the Kronecker delta, and $w_{0}$ is obtained by replacing $r$ in Eq. (5) by $r_{0}$. For functions orthogonal on the range 0,1 with unit weight function, equations equivalent to (15) and (17) are:

$$
\delta\left(r-r_{0}\right)=\sum_{j} b_{i j} R_{i j}\left(a_{j} r\right)
$$

and

$$
R_{i k}\left(a_{k} r_{0}\right)=b_{i k} .
$$

Comparison of results obtained from Eqs. (15) and (17) with those obtained from Eqs. (18) and (19) serves to illustrate the effects of weight function, Eq. (4), and change of variable, Eq. (5).

In Table I, peak heights are fairly consistent from $r_{0}=0.2$ to $r_{0}=0.8$. Half-widths are fairly consistent from $r_{0}=0.2$ to $r_{0}=0.9$. 
TABLE I $\sum_{j} b_{0 j} R_{0 j}\left(a_{j} r\right), j=1,2, \ldots, 17$ (Eqs. (18), (19))

\begin{tabular}{lccc}
\hline$r_{0}$ & Peak-height & Half-width & p.h. $\times$ h.w. \\
\hline 0.1 & 15.5 & .080 & 1.23 \\
0.2 & 17.5 & .069 & 1.21 \\
0.3 & 17.4 & .069 & 1.20 \\
0.4 & 16.5 & .073 & 1.20 \\
0.5 & 17.0 & .071 & 1.20 \\
0.6 & 17.5 & .069 & 1.21 \\
0.7 & 16.6 & .072 & 1.21 \\
0.8 & 16.5 & .073 & 1.20 \\
0.9 & 18.5 & .069 & 1.28 \\
\hline
\end{tabular}

TABLE II $\sum_{j} b_{i j} R_{0 j}\left(a_{j} w\right), j=1,2, \ldots, 17$ (Eqs. (15), (17))

\begin{tabular}{lccccccc}
\hline$r_{0}$ & $w_{0}$ & $P(r)$ & $p . h$. & h.w. & p.h. $\times$ h.w. & p.h. $/ P(r)$ & h.w. $(w)$ \\
\hline 0.1 & .221 & 2.06 & 33.4 & .036 & 1.20 & 18.9 & .074 \\
0.2 & .411 & 1.76 & 29.9 & .042 & 1.26 & 17.0 & .075 \\
0.3 & .573 & 1.47 & 24.2 & .050 & 1.20 & 16.5 & .073 \\
0.4 & .706 & 1.19 & 20.2 & .060 & 1.21 & 17.0 & .071 \\
0.5 & .811 & .921 & 14.9 & .082 & 1.22 & 16.1 & .075 \\
0.6 & .891 & .671 & 12.2 & .103 & 1.25 & 18.1 & .067 \\
0.7 & .946 & .443 & 8.46 & .176 & 1.49 & 19.1 & .067 \\
0.8 & .980 & .245 & 3.47 & .161 & .560 & 14.1 & .055 \\
\hline
\end{tabular}

(peaks are displaced for $r_{0}=0.7,0.8$ ).

The product of peak height $x$ half-width (a rough measure of peak area) is fairly consistent from $r_{0}=0.2$ to $r_{0}=0.9$. In Table II, the product of peak height $x$ half-width is fairly consistent from $r_{0}=0.1$ to $r_{0}=0.6$. Peak height divided by $P(r)$ is reasonably consistent with peak height in Table I from $r_{0}=0.2$ to $r_{0}=0.7$. h.w. $(w)$ was calculated by calculating $w$ coordinates corresponding to $r$ coordinates of half-height positions, and taking the difference between the resulting $w$ coordinates. The results are reasonably consistent with halfwidth values in Table I.

\section{CONCLUSIONS}

A series of functions, orthonormal on the range $r=0,1$, with weight function corresponding to the probability, $P(r)$, of finding a vector of length $r=|r|$ in a circle of unit diameter has been developed, by introducing a change of variable in spherical Bessel functions, 
orthogonal on the range $r=0,1$, with unit weight function. Principal effects of change of variable are reduction of peak half-width for small $r$, increase of peak half-width for large $r$, and increase of the argument of the functions, restricting their use to the approximate range $r=0.1$ to 0.6. (See Tabs. I and II and related discussion.)

\section{References}

Bunge, H. J. (1965) z.Metallkde., 56, 872-4.

Kröner, E. (1986) Statistical Modelling, In: Modeling Small Deformations of Polycrystals, Gittus, J. and Zarka, J. Eds., Elsevier Applied Science, London, pp. 229-91.

Landau, L. D. and Lifshitz, E. M. (1977) Quantum Mechanics (Non-relativistic Theory), Pergamon Press, Oxford, p. 107.

McLachlan, N. W. (1955) Bessel Functions for Engineers, Oxford, London, pp. 103-4.

Morris, P. R., Wang, T. T. and Adams, B. L. (1988) Two-Point Orientation-Coherence Functions, In: Eighth International Conference on Textures of Materials, Kallend, J. S. and Gottstein, G. Eds., Met. Soc., Warrendale, PA, pp. 157-68.

Roe, R. J. (1965) J. Appl. Phys., 36, 2024-31.

Wang, T. T. (1988) Ph.D. Dissertation, Evolution of the Two-Point Orientation Coherence Function in Plane-Strain Deformation, Mech. Eng. Dept., Brigham Young University, pp. 13-19. 